\title{
Unicompartmental Knee Arthroplasty: A Perspective from Computer Assisted Navigation
}

\author{
Asode Ananthram Shetty ${ }^{1}$, Vishvas Shetty ${ }^{1}$, Seok-Jung Kim ${ }^{2 *}$ \\ ${ }^{1}$ Faculty of Health and Social Sciences, Chatham Maritime, Canterbury Christ Church University, Kent, UK; ${ }^{2}$ Department of Ortho- \\ pedic Surgery, College of Medicine, Catholic University of Korea, Seoul, South Korea. \\ Email: *peter@catholic.ac.kr
}

Received April 24 $4^{\text {th }}, 2013$; revised May 25 ${ }^{\text {th }}, 2013$; accepted June $2^{\text {nd }}, 2013$

Copyright (C) 2013 Asode Ananthram Shetty et al. This is an open access article distributed under the Creative Commons Attribution License, which permits unrestricted use, distribution, and reproduction in any medium, provided the original work is properly cited.

\begin{abstract}
Unicompartmental Knee Arthroplasty (UKA) is an established procedure for the treatment of unicompartmental arthritis. Success depends on a clear understanding of the principles and kinematics of the knee. Restoration of the physiological axis and soft tissue balancing is the key to a successful outcome. We outline the basic principles of UKA and the role of computer assisted surgery in achieving these goals.
\end{abstract}

Keywords: Unicompartmental Knee Arthroplasty; Computer Assisted Navigation Surgery; Unicompartmental Arthritis

\section{Introduction}

Unicompartmental Knee Arthroplasty (UKA) was first introduced in the 1970s to treat unicompartmental arthritis and has since become a well-established surgical procedure. Impressive survival results are reported for medial compartment UKA [1,2]. These results, however, are confined to a few centres and prostheses (Norwegian register). There is considerable variation in the results from different centres and for different prostheses.

\section{Background}

The long term studies of UKA show a good correlation between 7 years survival rates and longer term outcomes [3]. The survival of modern implants has been improved by better implant design and improved polythene wear properties. The success of the UKA as well as Total Knee Replacement (TKA) is defined by 10-year survival. Although there is a $90 \% 10$-year survival rate, a number of studies have shown that functional results of UKA can be unsatisfactory. Up to $10 \%$ of patients express dissatisfaction with the functional outcome. Technical error seems to be the main reason for poor functional outcome and premature failure [2,4]. Poor soft tissue balance, flexion-extension mismatch, and malalignment may be the factors responsible.

The primary goal of knee replacement is to implant the

${ }^{*}$ Corresponding author. prosthesis accurately in order to restore sagittal, coronal and axial limb alignment. The introduction of minimally invasive surgery (MIS), aimed at reducing soft tissue trauma and blood loss and to aid rapid postoperative recovery, has made this goal challenging. Better instrumentation evolved to accommodate MIS unicompartmental knee arthroplasty. Despite these advances, open UKA is not as accurate as open TKA in restoring limb alignment and implant position. This alignment deteriorates further in MIS UKA [5].

Computer assisted navigation surgery can significantly improve the restoration of limb alignment and implant position, by increasing the precision of the bony cuts, and improving soft tissue balancing and knee kinematics [6]. Computer assisted navigation knee surgery is an interactive operative monitoring system designed to improve the surgical performance and clinical outcome of the knee replacement. It facilitates accurate decision making for alignment and orientation of instruments, trials and ultimately the implant. The system also provides surgeons with preoperative, intraoperative and post-implantation assessments of the patient's joint kinematics and soft tissue balancing. It is assumed that optimal soft tissue balancing will improve joint stability and may reduce the need for prolonged rehabilitation. MIS UKA may be on the brink of a major advance through the achievement of better technical alignment using computer navigation [6].

The navigation system uses the mechanical axis to compute the varus/valgus, flexion/extension and internal/ 
external rotation of the component. It also analyses the medial and lateral ligament lengthening behaviour throughout the range of motion to achieve optimum soft tissue balance.

\section{Concept of Knee Axis (Figure 1)}

It is important to understand the concept of physiological axis and zero degree mechanical axis in knee arthroplasty. In TKA, the mechanical axis (Figure 1(c)) passes through the centre of the hip rotation, centre of the knee and the ankle centre. The joint line is horizontal and both medial and lateral compartments are equally loaded [7].

In a normal healthy knee, however, the mechanical load is predominantly medial, a reflection of the greater surface area for weight bearing compared to the lateral side [8] (Figure 1(a)). The object of UKA is to restore the mechanical axis of the lower limb to its physiological position (femoro-tibial segmental axis) before the onset of degenerative changes (Figure 1(b)). This is the concept of physiological axis of the unicompartmental knee, which differs significantly from the rationale of zero de gree mechanical axis restoration in TKA [9]. Studies of normal populations have shown the physiological femoro-tibial segmental axis is slightly varus $[10,11]$.

\section{Concept of Soft Tissue Envelope (Figure 2)}

UKA is primarily a resurfacing and ligament balancing procedure without ligament release. Hence, it is important to understand the pathology of varus and valgus arthritis. Varus arthritis is primarily an extension disease affecting the distal femur; valgus arthritis is a flexion disease with degenerative changes the posterior femoral condyle. This has a bearing on the soft tissue balancing as the different disease patterns affect the flexion and extension gap, and rotation of the component in placements.

Computer assisted surgery enables the accurate location of bony landmarks in the lower limb from which pre resection alignment can be ascertained. Simultaneously, ligament laxity can be determined in the full range of motion. Appropriate and accurate bony resection is then performed to correct the deformity and restore the physiological axis and ligament balance without soft-tissue release.

In most patients with a varus deformity, this results in a slightly varus knee, which is often referred to as slightly undercorrected in relation to the mechanical axis. This restores the patient to their normal varus alignment (physiological axis). During navigation surgery, intraoperative data feedback helps to restore this patientspecific axis accurately.

Failure to correct the physiological axis in some varus
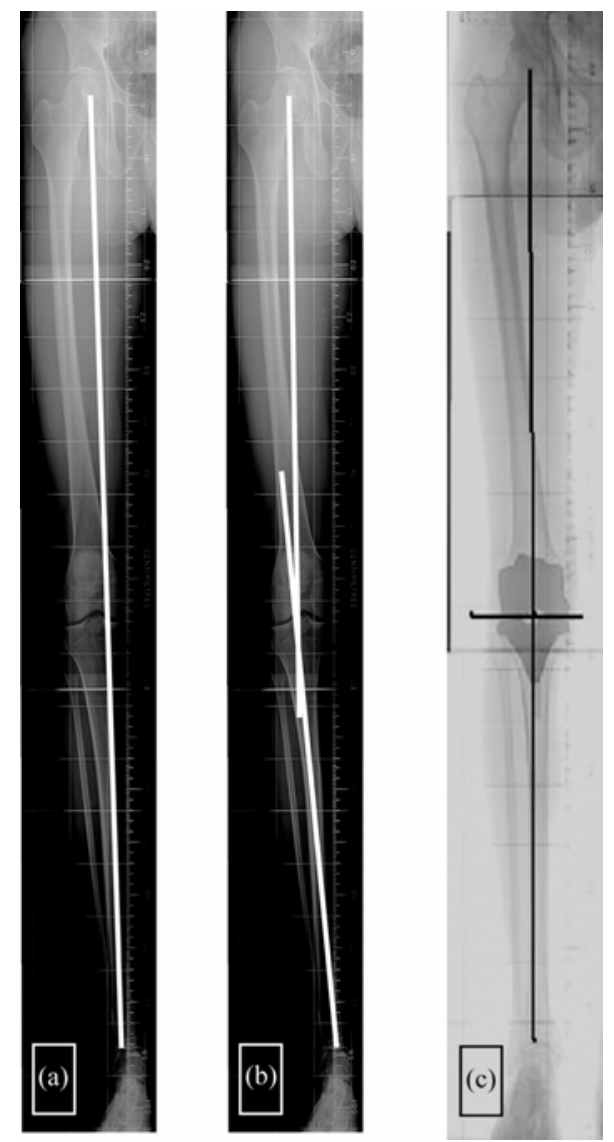

Figure 1. (a) Mechanical axis; (b) Physiological axis (segmental); (c) 0 degree mechanical axis of total knee.

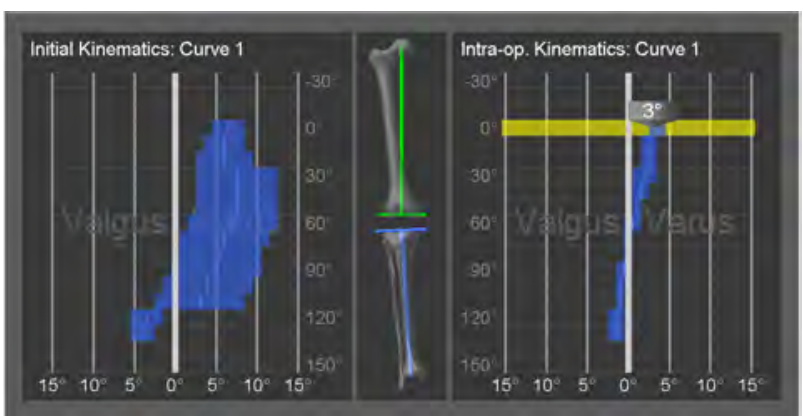

Figure 2. Computer navigated screenshots illustrating the soft tissue envelope in range of flexion: initial soft tissue laxity (left) and laxity after correction intra-operatively (right) mimicking 3 degree physiological axis.

results in a poor outcome. Over-correction of the axis results in overloading of the healthy lateral compartment and subsequent poor result (Figure 3). Under-correction of the axis leads to instability, or dislocation of the tibial insert in mobile bearing implants.

\section{Conclusions}

UKA is not "half a TKA". UKA is primarily a soft tissue balancing procedure with the restoration of a patient spe- 

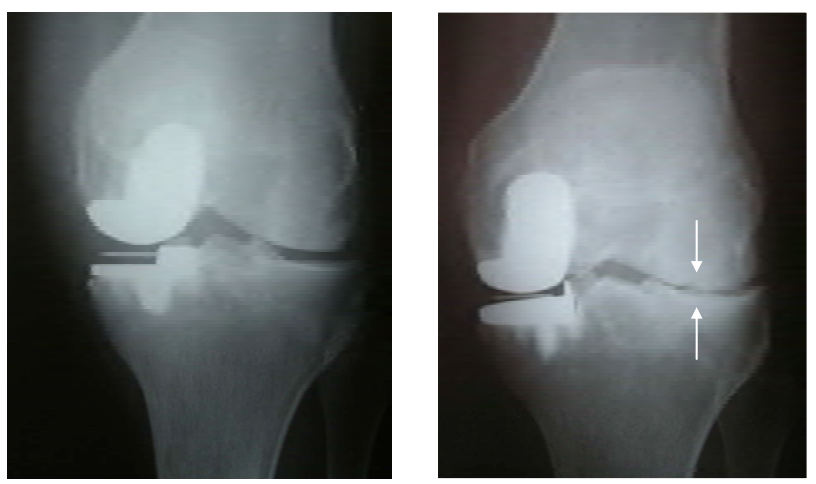

Figure 3. Over-correction leading to early lateral compartment wear (arrows).

cific physiological axis. In TKA varus-valgus alignment is determined by the distal femoral and proximal tibial cut. In UKA, however, because it is a resurfacing procedure, insert thickness determines the varus-valgus angle. Computer assisted surgery has the capacity to quantitatively assess, and helps to balance, the soft tissue tension intra-operatively allowing selection of the appropriate insert thickness. Accurate determination and restoration of the desired physiological limb axis and improved implant positioning allow ideal kinematics to be achieved.

A computer assisted system can improve the accuracy of standard instrumentation and enhance 3D perception and intra-operative orientation, especially in MIS. It provides accurate quantitative data of bone resection, alignment and kinematics. This should eliminate the error of "eyeballing".

Studies of computer assisted surgery have shown improved radiological alignment and functional results $[12,13]$. It is well accepted that mal-alignment will lead to early failure and so, logically, better alignment should lead to superior functional results with long-term survival.

The potential for better results using computer surgery represents a great advance for UKA surgery. This should encourage more surgeons to consider UKA as an alternative to TKA in patients for whom there are no contraindications.

\section{REFERENCES}

[1] A. J. Price, et al., "Ten-Year in Vivo Wear Measurement of a Fully Congruent Mobile Bearing Unicompartmental Knee Arthroplasty,” The Journal of Bone \& Joint Surgery, Vol. 87, No. 11, 2005, pp. 1493-1497. doi:10.1302/0301-620X.87B11.16325
[2] D. W. Murray, J. W. Goodfellow and J. J. O’Connor, "The Oxford Medial Unicompartmental Arthroplasty: A Ten-Year Survival Study," The Journal of Bone \& Joint Surgery, Vol. 80, No. 6, 1998, pp. 983-989. doi:10.1302/0301-620X.80B6.8177

[3] U. C. Svard and A. J. Price, "Oxford Medial Unicompartmental Knee Arthroplasty. A Survival Analysis of an Independent Series," The Journal of Bone \& Joint Surgery, Vol. 83, No. 2, 2001, pp. 191-194. doi:10.1302/0301-620X.83B2.10966

[4] J. L. Rees, et al., "Minimally Invasive Oxford Unicompartmental Knee Arthroplasty: Functional Results at 1 Year and the Effect of Surgical Inexperience,” Knee, Vol. 11, No. 5, 2004, pp. 363-367. doi:10.1016/j.knee.2003.12.006

[5] D. A. Fisher, M. Watts and K. E. Davis, "Implant Position in Knee Surgery: A Comparison of Minimally Invasive, Open Unicompartmental, and Total Knee Arthroplasty,” Journal of Arthroplasty, Vol. 18, Suppl. 1, 2003, pp. 2-8. doi:10.1016/S0883-5403(03)00291-2

[6] G. Keene, D. Simpson and Y. Kalairajah, "Limb Alignment in Computer-Assisted Minimally-Invasive Unicompartmental Knee Replacement,” The Journal of Bone \& Joint Surgery, Vol. 88, No. 1, 2006, pp. 44-48. doi:10.1302/0301-620X.88B1.16266

[7] H. P. Hsu, et al., "Effect of Knee Component Alignment on Tibial Load Distribution with Clinical Correlation," Clinical Orthopaedics and Related Research, Vol. 248, 1989, pp. 135-144.

[8] D. B. Kettelkamp and A. W. Jacobs, “Tibiofemoral Contact Area-Determination and Implications,” The Journal of Bone \& Joint Surgery, Vol. 54, No. 2, 1972, pp. 349-356.

[9] P. Cartier, “Unicompartmental Knee Arthroplasty,” 1997.

[10] D. Paley and K. Tetsworth, "Mechanical Axis Deviation of the Lower Limbs. Preoperative Planning of Multiapical Frontal Plane Angular and Bowing Deformities of the Femur and Tibia," Clinical Orthopaedics and Related Research, Vol. 280, 1992, pp. 65-71.

[11] R. W. Hsu, et al., "Normal Axial Alignment of the Lower Extremity and Load-Bearing Distribution at the Knee," Clinical Orthopaedics and Related Research, Vol. 255, 1990, pp. 215-227.

[12] L. Perlick, et al., "Minimally Invasive Unicompartmental Knee Replacement with a Nonimage-Based Navigation System,” International Orthopaedics, Vol. 28, No. 4, 2004, pp. 193-197.

[13] J. Y. Jenny and C. Boeri, "Unicompartmental Knee Prosthesis Implantation with a Non-Image-Based Navigation System: Rationale, Technique, Case-Control Comparative Study with a Conventional Instrumented Implantation,” Knee Surgery, Sports Traumatology, Arthroscopy, Vol. 11 , No. 1, 2003, pp. 40-45. 